\title{
Laser scanned photodiodes (LSP) for Image sensing
}

\author{
${ }^{\mathrm{I}}$ M. Vieira, ${ }^{1} \mathrm{M}$. Fernandes, ${ }^{\text {P }}$. Louro, ${ }^{\mathrm{I}}$ R. Schwarz, M. Schubert \\ ${ }^{1}$ Electronics and Communications Dept., ISEL, R. Conselheiro Emídio Navarro, P 1949-014 Lisboa, \\ Portugal. Tel: +35121 8317181, Fax:+351218317114, E-mail: mv@isel.pt. \\ ${ }^{2}$ Institut fur Physikalische Elektronik, Universitat Stuttgart, Pfaffenwaldring 47, D-70569, Stuttgard, \\ Germany
}

\begin{abstract}
SUMMARY
An optimized $\mathrm{ZnO}: \mathrm{Al} / \mathrm{a}$-pin $\mathrm{Si}_{\mathrm{x}} \mathrm{C}_{1-\mathrm{x}}: \mathrm{H} / \mathrm{Al}$ configuration for the laser scanned photodiode (LSP) imaging detector is proposed. The LSP utilizes light induced depletion layers as detector and a laser beam for readout.

The effect of the sensing element structure, cell configuration and light source flux are investigated and correlated with the sensor output characteristics. Experimental data reveal that the large optical gap and the low conductivity of the doped $\mathrm{a}-\mathrm{Si}_{\mathrm{x}} \mathrm{C}_{\mathrm{l-x}}: \mathrm{H}$ layers are responsible by an induced inversion layer at the illuminated interfaces which blocks the carrier collection. These insulator-like layers act as MIS gates preventing image smearing. The physical background of the LSP is discussed.
\end{abstract}

Keywords: Laser scanner photodiode, image acquisition and representation, analog readout.

\section{Introduction}

Image sensors used in commercial devices include photodiode arrays $[1,2]$ charge-coupled device (CCD) [3, 4], complementary metal oxide semiconductor (CMOS) [5], charge injecting devices (CID) [6], and bipolar transistors [7]. All of these devices use essentially the same light sensing mechanism. Photons penetrating a depletion region generate electron-hole pairs. These carriers are swept away by the electric field across the depletion region and generate a small transverse photocurrent that is used for image sensing.

In the past we have developed a different type of image sensor, the Laser Scanned Photodiode (LSP) sensor [8]. This sensor is fundamentally different from the conventional ones since it is based on one single sensing element and uses a modulated, low-power laser beam to scan and acquire the image directly.

Our recent work on a-Si:H $\mathrm{p}-\mathrm{i}-\mathrm{n}$ LSP sensors have shown that replacing the a-SiH n-layer with a high resistive and weakly doped a-SiC: $\mathrm{H}$ layer results in a remarkable improvement in the sensor spatial resolution [9]. This improvement was ascribed to an enhancement of the electric field modulation across the depletion region that prevents the carriers to smear out in the lateral direction [9]. In this work a detailed analysis of this optimized structure is given. The physical background of the LSP is presented. The effects of the sensor structure and image brightness on the output characteristics are discussed.

\section{The physics of the p-i-n photodiode under non uniform illumination}

A light pattern projected onto a a-Si:H based $p-i-n$ photodiode leads to a distortion of space charge regions at the junctions giving rise to a modulation of the potential barrier $[10,11]$. Low local potential barriers are ascribed to illuminated regions and high potential barriers to dark zones [12]. If an a-SiC:H/a-Si:H heterojunction is used the band misalignment leads to the high accumulation of photogenerated carriers at the illuminated interfaces resulting in a high magnitude of the heterojunction barrier modulation. As a consequence, depending on the light flux intensity, the depletion region shrinks and the electric field is reduced. Low local or reversed electric fields are ascribed to illuminated regions and high local electric fields to dark zones. This effect is the basis of the Laser Scanned Photodiode (LSP) image sensor.

\section{Results and discussion}

\section{Laser Scanned Photodiode (LSP)}

The LSP sensor is a two dimensional structure composed of a p-i-n photodiode (the active element) and transparent front and metal back contacts. The physical structure of a Laser Scanned Photodiode (LSP) under a light pattern illumination is displayed in Fig. I.

To optically map the image, a steady-state light pattern is projected through the transparent contact onto a fixed location of the photosensitive surface. For image acquisition, a low-power chopped laser spot scans the sensor in the raster mode. The read-out of the injected carriers is achieved by measuring the $a c$ component of the short circuit current, $I_{s c}$. The image intensity, $X_{m, n}$, is obtained by subtracting the input matrix $I_{m, n}$ (with image) from the background, $b_{m, n}$ (without image). It was 
observed that the signal-to-noise ratio $(\mathrm{S} / \mathrm{N})$ depends on the relationship between the light source fluxes of the scanner $\left(\Phi_{\mathrm{S}}\right)$, and the image $\left(\Phi_{\mathrm{L}}\right)$ for a constant value of the n-layer conductivity [9].

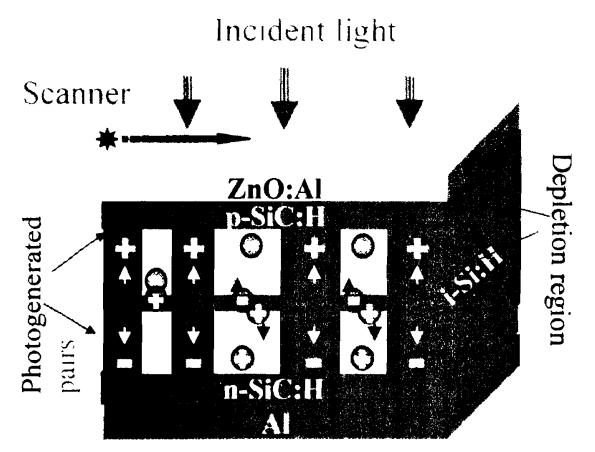

Fig.1: Physical structure of a LSP sensor under a light pattern illumination. The depletion region modulation and the carrier movement are superimposed.

LSP optical-to-electrical transfer characteristics

Large area $\left(4 \times 4 \mathrm{~cm}^{2}\right)$ image transducers in a glass/ZnO:Al/p $\left(\mathrm{Si}_{\mathbf{x}} \mathrm{C}_{1-\mathrm{x}}: \mathrm{H}\right) / \mathrm{i}(\mathrm{Si}: \mathrm{H}) / \mathrm{n}\left(\mathrm{Si}_{\mathrm{x}} \mathrm{C}_{1-\mathrm{x}}: \mathrm{H}\right) / \mathrm{Al}$

configuration were produced. The $\mathrm{p}-\mathrm{i}-\mathrm{n}$ transducer layers were deposited at low temperature $\left(110^{\circ} \mathrm{C}\right)$ by Plasma Enhanced Chemical Vapor Deposition at $13.56 \mathrm{MHz}$ [13]. The front and back contacts were produced by sputtering and thermal evaporation, respectively. The deposition conditions where kept constant for all i-layers, while they varied in the doped layers $(0.5<x<1)$. In order to infer the electro-optical properties of each single layer, intrinsic and doped a-SiC:H films were deposited on Corning glass AF45 under the same conditions. Al the layers on sample \#M006291 are based in amorphous hydrogenated silicon (a-Si:H), while the p-layer in \#M006301 and the p- and n-layers in \#M007192 sensors are based on a-SiC:H alloy.

TableI Optoelectronic properties of the individual layers.

\begin{tabular}{|c|c|c|c|c|c|}
\hline Cell code & & $\begin{array}{c}\sigma_{\mathrm{d}} \\
\left(\Omega^{-1} \cdot \mathrm{cm}^{-}\right. \\
1)\end{array}$ & $\begin{array}{c}\Delta \mathrm{E} \\
(\mathrm{eV})\end{array}$ & $\begin{array}{c}\mathrm{E}_{\mathrm{op}} \\
(\mathrm{eV})\end{array}$ & $\sigma_{\mathrm{ph}} / \sigma_{\mathrm{d}}$ \\
\hline \multirow{3}{*}{ \#006291 } & $\mathrm{p}$ & $8.2 \times 10^{-7}$ & 0.499 & 1.80 & 7.3 \\
& $\mathrm{i}$ & $7.6 \times 10^{-11}$ & 0.739 & 1.79 & $7.1 \times 10^{4}$ \\
& $\mathrm{n}$ & $1.8 \times 10^{-5}$ & 0.426 & 1.82 & 7.0 \\
\hline & $\mathrm{p}$ & $8.2 \times 10^{-7}$ & 0.499 & 1.80 & 7.3 \\
\#M006301 & $\mathrm{i}$ & $7.6 \times 10^{-11}$ & 0.739 & 1.79 & $7.1 \times 10^{4}$ \\
& $\mathrm{n}$ & $1.9 \times 10^{-12}$ & 0.834 & 2.10 & 21 \\
\hline & $\mathrm{p}$ & $2.5 \times 10^{-9}$ & 0.649 & 2.06 & 4.5 \\
\#M007192 & $\mathrm{i}$ & $7.6 \times 10^{-11}$ & 0.739 & 1.79 & $7.1 \times 10^{4}$ \\
& $\mathrm{n}$ & $1.9 \times 10^{-12}$ & 0.834 & 2.10 & 21 \\
\hline
\end{tabular}

A preliminary electrical and optical characterization of the films was carried out by measuring the electrical conductivity in the coplanar direction and by obtaining the absorption spectra from transmission and reflection measurements. The front $\mathrm{ZnO}: \mathrm{Al}$ contact is $300 \mathrm{~nm}$ thick has a transmissivity of $80 \%$ (from $425 \mathrm{~nm}$ to $700 \mathrm{~nm}$ ), and a resistivity around $9 \times 10^{-4} \Omega \mathrm{cm}$. The dark conductivity, $\sigma_{d}$, and the photoconductivity, $\sigma_{p h^{\prime}}$ under AM1.5 $\left(100 \mathrm{~mW} / \mathrm{cm}^{2}\right)$, the activation energy, $\Delta \mathrm{E}$, and the optical Tauc gap, $E_{o p}$, for the analyzed sensors are summarized in Table I.

All the structures were characterized by current-voltage and capacitance-voltage measurement in dark and under illumination.

To confirm the physical model displayed in Fig. 1 we have determined the capacitance-voltage characteristics (\#M006301) under different illumination conditions (Fiig.2) and have inferred the depletion width, w, at zero applied bias.

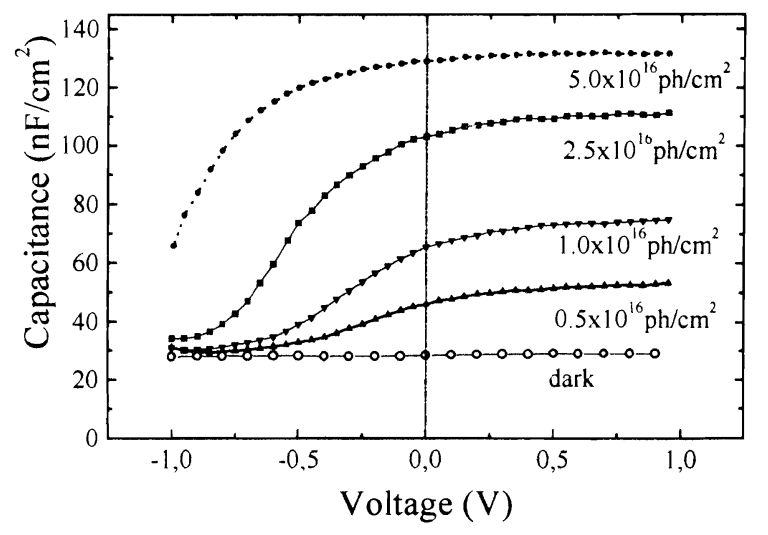

Fig. 2: C-V characteristic under different light intensities.

Results show that the capacitance increases with the light intensity. In the dark and under zero bias $\mathrm{V}=0$, the device is fully depleted $(330 \mathrm{~nm})$ while under $20 \mathrm{~mW} / \mathrm{cm}^{2}$ the depletion width decreases to values around $56 \mathrm{~nm}$, suggesting a change in the current collection from a fieldaided drift to a diffusion controlled mechanism. In the heterojunctions the higher optical gaps and the lower conductivity of the doped $\mathrm{a}-\mathrm{Si}_{\mathbf{x}} \mathrm{C}_{1-\mathrm{x}}: \mathrm{H}$ layers, when compared either with the intrinsic a-Si:H or even with the doped layers in the homostructure, are responsible for an induced inversion layer at the illuminated interfaces which will block the carrier collection (Fig.1). These insulatorlike layers act as MIS gates that prevent excess signal charge from spreading to the nearby dark regions, which could lead to image smearing.

\section{Sensor Element Performance}

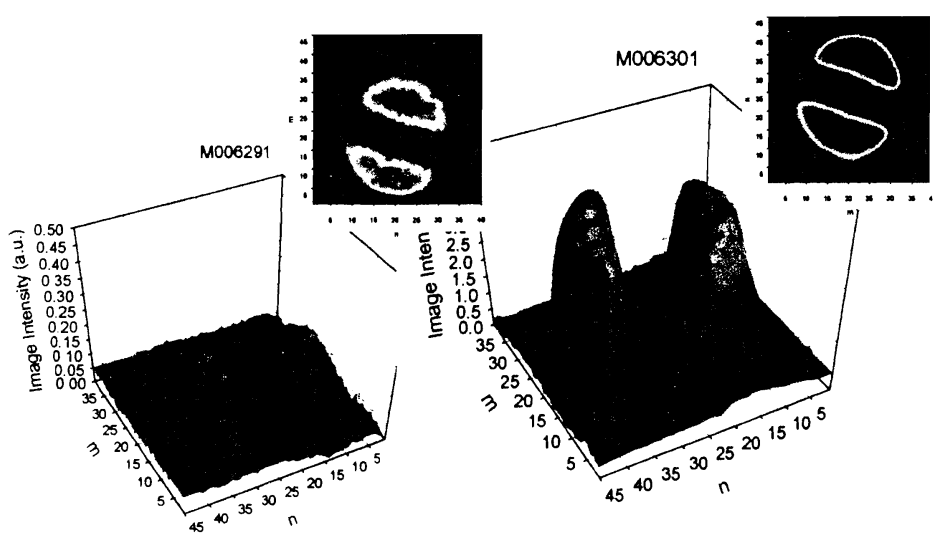

Fig. 3: Image intensity and grey level for sensors with differently doped n-layer conductivity. 
In Fig. 3 the image signal of a circle of $6 \mathrm{~mm}$ diameter cut into two halves by a $2 \mathrm{~mm}$ gap is displayed for two transducers (\#M006291, \#M006301) having different doped layer conductivities. By using highly resistive doped layers (\# M006301) a good image representation is achieved with sharp edges and almost no blur. If the doped layer conductivity is higher (\#M006291), the image intensity decreases and spreads out in the lateral direction. Results show that the observed differences in the resolution depend on n-layer conductivity for a constant value of the p-layer resistivity and light flux intensity. The image signal and the spatial resolution are increased when the n-layer conductivity decreases. The resolution in the sensor with the most resistive n-layer was less than $50 \mu \mathrm{m}$ with a $\mathrm{S} / \mathrm{N}$ ratio of the order of $32 \mathrm{~dB}$.

This behavior can be explained through the lateral photoeffect [12] that allows the carriers to drift across the doped layers towards the nearby dark regions. If the conductivity of the doped layer is high the depletion layer modulation across the junction decreases due to the increase of the ratio between the lateral and transverse electrical field. Some of the carriers generated by the scanner inside the depletion regions drift along the doped layers towards the illuminated region (lower potential barriers), and are not collected leading to a lower image signal and to some blur. If a highly resistive and wide gap n-layer is used the collection and/or recombination occurs mainly beneath the generation point since the carrier flows in the transverse direction. The lateral photo-effect is minimized, resulting in an increased spatial resolution.

In Fig. 4 the image intensity as a function of light flux for the \#M006301 sensor is displayed and in Fig.5 the capacitance and the depletion width dependence with $\Phi_{\mathrm{L}}$ (uniform illumination) are shown. During the image acquisition process, the scanner intensity was kept constant while the light source flux used for mapping the image onto the sensor was changed $\left(10^{-2} \mathrm{~mW} / \mathrm{cm}^{2}\right.$ $<\Phi_{\mathrm{L}}<20 \mathrm{~mW} / \mathrm{cm}^{2}$ )

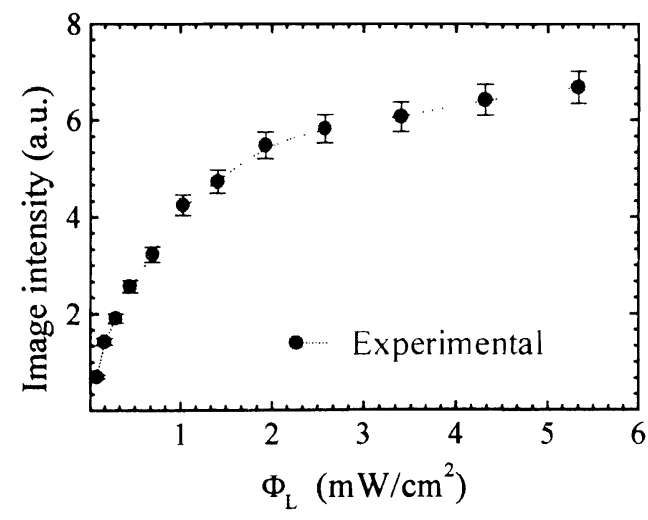

Fig. 4: Image intensity as a function of light flux.

Results show that as $\Phi_{\mathrm{L}}$ increases, the image signal increases, reaching a saturation value at $\Phi_{\mathrm{L}}>4 \mathrm{~mW} / \mathrm{cm}^{2}$. The sensor presents a good linearity at low signal levels and starts to saturate at around $70 \%$ of maximum value. The minimum detectable light flux is of the order of 0.2 $\mathrm{mW} / \mathrm{cm}^{2}$.
It is interesting to note that the image intensity and the capacitance at zero bias present the same trend with light flux, confirming the proposed model. As $\Phi_{\mathrm{L}}$ increases, the depletion region shrinks initially in a linear way reaching its minimum value when the flat band condition is achieved. The image signal will increase with $\Phi_{\mathrm{L}}$ and saturates for the minimum value of the depletion width. During the image acquisition process, only the carriers generated by the scanner within the depletion region are swept away by the existing electric field and are collected, otherwise they diffuse, recombine or are trapped in the bulk.

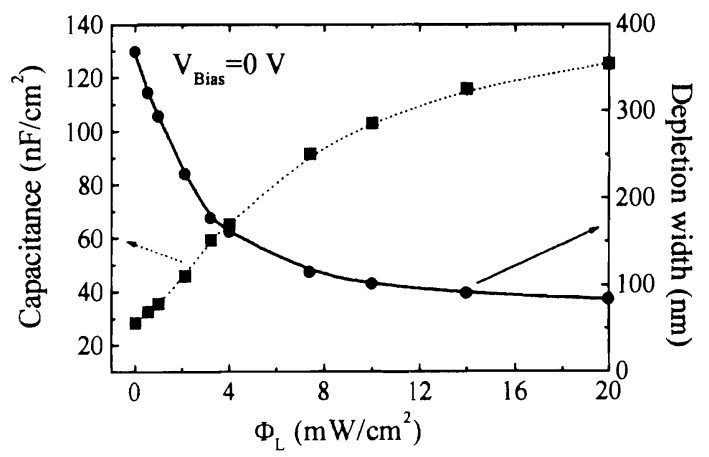

Fig.5: Capacitance and depletion width dependence with $\Phi_{L}$ (uniform illumination).

For a test of the sensor responsivity a $7.5 \mathrm{~mm}$ donut-like image with an inner hole of $2.5 \mathrm{~mm}$ diameter was projected onto the sensor with the most resistive n-layer (\# M006301). Fig. 6 display the acquired images, respectively, at $\Phi_{\mathrm{L}}=5 \mathrm{~mW} / \mathrm{cm}^{2}$ and $\Phi_{\mathrm{L}}=0.5 \mathrm{~mW} / \mathrm{cm}^{2}$, keeping $\Phi_{\mathrm{S}}=2.5 \times 10^{-2} \mathrm{~mW} / \mathrm{cm}^{2}$.
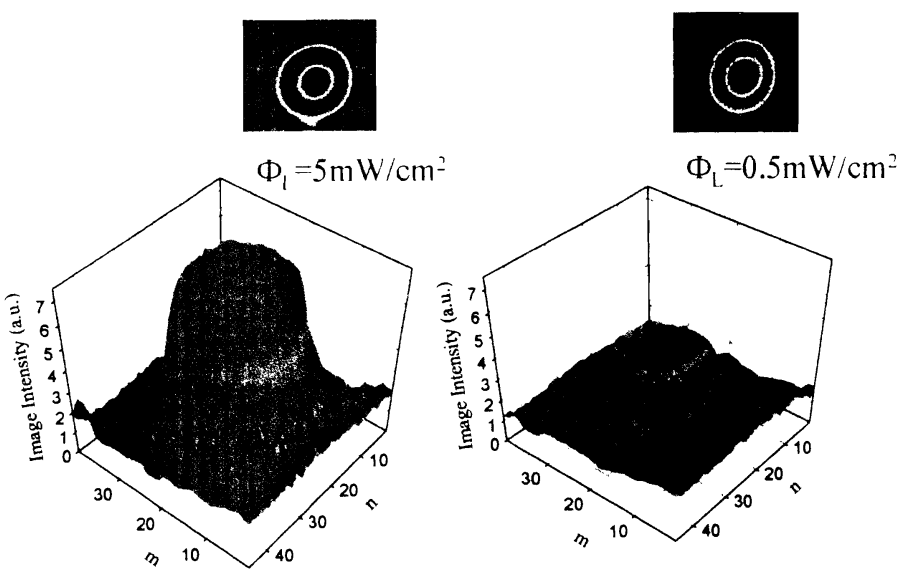

Fig.6: Image intensity and grey levels keeping $\Phi_{S}=2.5 \times 10^{-2} \mathrm{~mW} / \mathrm{cm}^{2}$.

The results show a good contrast between the illuminated and the dark regions even at low flux. For highly resistive n-layers the carriers injected by the scanner flow mainly in the transversal direction. Nevertheless, if the light source flux is high enough (saturation condition) the excess charge carriers generated from the bright part of the image spread out giving rise to some blooming at the edges. The saturation of the image signal is then limited by the ability 
of the depletion region to hold and to separate the photocarriers generated by the scanner. This depends on $\Phi_{\mathrm{L}}$ and on the junction electric field strength.

\section{Conclusions and future trends}

An optical $\mathrm{ZnO}: \mathrm{Al} / \mathrm{a}-$ pin $\mathrm{Si}_{\mathbf{x}} \mathrm{C}_{1-\mathrm{x}}: \mathrm{H} / \mathrm{Al}$ imager which uses a low power scanning beam to readout the photogenerated carriers was presented. The essence of the detection scheme is the analogue readout with the absence of semiconductor arrays or electrode potential manipulation to transfer the information coming from the transducer.

The results show that the responsivity, the linearity and the resolution are limited by the device structure, conductivity of the doped layers and by the light flux used to map the image onto the sensor. An enhancement of magnitude in the image signal and in the spatial resolution are achieved by decreasing the n-layer conductivity.

Examples of possible applications for our optical sensor are medical imaging and display devices. Optical character recognition, facsimile transmitters and optical input point of sale terminals can also be foreseen. As a possible application in Fig. 7 we display the black and

\section{References}

[1] J. W. Horton, R. V. Mazza, and H. Dym, The scanistor-A solid state image sensor Proc. IEEE, 52 (1964) 1513-1519.

[2] P. W. Fry, Silicon photodiode arrays, J. of Physics E: Scientific Instruments, 8 (1975) 337349.

[3] G. F. Amelio, Charge coupled devices, Scientifc American, 230 (1974) 22-31.

[4] S. Chakrabarti, O. H. W. Siegmund, and J. Hecht, Continous readout photon counting imaging detectors, SPIE 834 (1987) 222-226.

[5] Decker, Steven, A 256x256 CMOS imaging array with wide dynamic range pixels and columnparallel digital output, IEEE Journal of Solid State Circuits [H.W. Wilson - AST] Vol. 33 (1998), Iss. 12; 2081-2083.

[6] H. K. Burke and G. J. Michon, Charge injection imaging: operating techniques and performance characteristics, IEEE Trans. Electron Dev. ED 23, (1976) 189-195.

[7] M. A. Schuster, and G. Strull, A monolithic mosaic of photon sensor for solid state imaging applications, IEEE Trans. Electron Dev. ED 13 (1966) 907-1001.

[8] F. Sousa, J. Martins, M. Fernandes, A. Maçarico, R. Schwarz, and M. Vieira, Image processing in a $\mu c-S i: H$-i- $n$ transducer, J. of Non-Crystalline Solids, 266-269 (2000) 1228-1232.

[9] M. Vieira, M. Fernandes, J. Martins, P. Louro, A. Maçarico, R. Schwarz, and M. Schubert, Improved Resolution in a $p-i-n$ Image Sensor by Changing the Structure of the Doped Layers white image of a text word (ISEL) and a greyscale photo representation obtained with the transducer \#M006301. No image processing algorithms were used.

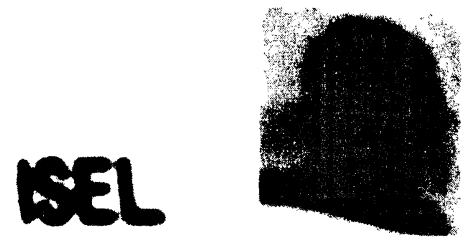

Fig. 7: Black and white image of a text word (ISEL) and a grayscale photo representation.

\section{Acknowledgements}

We would like to thank IPE for helpful discussions concerning this study. This work has been financially supported by PRAXIS/P/EEI/12183/1998, and by INIDA projects.

Mat. Res. Soc. Symp. Proc. (S. Francisco, USA, April 2000) to be published.

[10] J.T.Wallmark, A new semiconductor photocell using lateral photoeffect, Proc. IRE 43 (1956) 474-483.

[11] M. Vieira, Speed photodetectors based on amorphous and microcrystaliine silicon $p-i-n$ devices, Appl. Phys. Lett. 70 (1997) 220.

[12] M. Vieira, S. Koynov, A. Fantoni , and R. Schwarz, Wide Spectral response in $\mu \mathrm{c}-\mathrm{Si}: \mathrm{H}$ photodiodes, Thin Solid Films 296 (1997) 164169.

[13] C. Koch, M. Ito, M. Schubert, and J. H. Werner, Low-Temperature Deposition of Amorphous Silicon Based Solar Cells, Mat. Res. Soc. Symp. Proc., 575 (1999) 749-755. 